\title{
Cerebrospinal Fluid S100B and Alzheimer's Disease Biomarkers in Hip Fracture Patients with Delirium
}

\author{
Karen Roksund Hov ${ }^{a, b}$ Nils Bolstad ${ }^{b, c}$ Ane-Victoria Idland ${ }^{a, b}, d$ \\ Henrik Zetterberg ${ }^{\text {e, } f, g}$ Kaj Blennow ${ }^{\text {ef }}$ Farrukh A. Chaudhry ${ }^{c, h}$ \\ Frede Frihagen $^{i} \quad$ Johan Ræder ${ }^{b}, j$ Torgeir Bruun Wyller ${ }^{a, b}$ \\ Leiv Otto Watne ${ }^{a, h}$

\begin{abstract}
a Oslo Delirium Research Group, Department of Geriatric Medicine, Oslo University Hospital, Oslo, Norway; ${ }^{b}$ Institute of Clinical Medicine, University of Oslo, Oslo, Norway; 'Department of Medical Biochemistry, Oslo University Hospital, Oslo, Norway;

${ }^{\mathrm{d}}$ Research Group for Lifespan Changes in Brain and Cognition, Department of Psychology, University of Oslo, Oslo, Norway; ${ }^{e}$ Department of Psychiatry and Neurochemistry, Institute of Neuroscience and Physiology, The Sahlgrenska Academy, University of Gothenburg, Mölndal, Sweden; ${ }^{f}$ Clinical Neurochemistry Laboratory, Sahlgrenska University Hospital, Mölndal, Sweden; 9Department of Molecular Neuroscience, UCL Institute of Neurology,

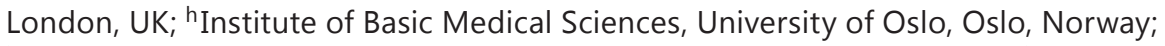
'Division of Orthopedic Surgery, Oslo University Hospital, Oslo, Norway; ${ }^{j}$ Department of Anesthesiology, Oslo University Hospital, Oslo, Norway
\end{abstract}

\section{Keywords}

Delirium $\cdot$ S100B $\cdot$ Cerebrospinal fluid $\cdot$ Phosphorylated tau $\cdot$ Hip fracture

\begin{abstract}
Objectives: This study aimed to investigate the relationship between cerebrospinal fluid (CSF) S100B astrocyte-derived protein and delirium and to perform stratified analyses according to clinical and CSF markers of dementia. Methods: We performed a prospective cohort study in a university hospital setting. The participants were patients admitted for hip fracture $(n=98)$ or for elective surgery $(n=50)$. Delirium was assessed daily perioperatively in hip fracture patients using the Confusion Assessment Method. A consensus-based diagnosis of prefracture dementia was made using all available information. CSF was drawn at the onset of spinal anesthesia. S100B and phosphorylated tau (P-tau) concentrations were measured using electrochemiluminescence immunoassay and enzyme-linked immunosorbent assays, respectively. Results: In the hip fracture population $(n=98)$ there was no significant difference in CSF S100B concentrations between patients with ongoing preoperative (i.e., prevalent) delirium ( $n=36$, median [interquartile range] $1.11 \mu \mathrm{g} / \mathrm{L}$ [0.91-1.29]) and patients who never developed delirium ( $n=46,1.08 \mu \mathrm{g} / \mathrm{L}$ [0.92-1.28], $p=0.92)$. In patients without preoperative delirium,
\end{abstract}


those who developed delirium postoperatively (i.e., incident delirium) $(n=16,1.38 \mu \mathrm{g} / \mathrm{L}[1.08-$ 1.62]) had higher concentrations of $\mathrm{S} 100 \mathrm{~B}$ than the 46 who never did ( $p=0.013$ ). This difference was confined to patients with pathological concentrations of P-tau ( $\geq 60 \mathrm{ng} / \mathrm{L}, n=38$ ). We also found that P-tau and S100B were correlated in CSF in the elective surgery patients. Conclusions: CSF S100B was elevated in patients with incident delirium who also had pathological levels of the Alzheimer disease biomarker P-tau, suggesting vulnerability caused by a preexisting process of astrocytic activation and tau pathology.

(C) 2017 The Author(s)

Published by S. Karger AG, Basel

\section{Introduction}

Delirium is a disturbance in attention, awareness, and cognition with acute onset. It is a direct physiological consequence of an acute medical condition, e.g., a hip fracture, and constitutes a common [1] and serious [2] complication of hospitalization. The pathophysiology is poorly understood $[3,4]$, and studies of cerebrospinal fluid (CSF) and serum biomarkers may elucidate pathophysiological mechanisms. Dementia is a major risk factor for delirium [5], and delirium is a strong predictor of both new-onset dementia [6] and acceleration of existing cognitive decline [7]. The emerging literature explores possible mechanisms [8], but still the details of the neuropathological links between dementia and delirium are not fully understood.

The calcium-binding protein S100B is expressed primarily [9], but not solely [10], by astrocytes within the CNS. Physiological concentrations are considered trophic to neuronal tissue, while higher concentrations may have toxic effects [11]. Serum concentrations of S100B have been studied in a number of conditions of brain pathology, and several studies have shown that elevated S100B concentration in serum is associated with delirium [12-14]. Results are, however, conflicting [15], probably partly because S100B in serum may derive also from extracerebral sites, such as fractures [16]. CSF S100B in hip fracture patients with delirium has been investigated in two studies. One study [17] found elevated CSF S100B in patients with prevalent delirium, whereas the other one could not reproduce this finding [18]. Moreover, one Australian study [19] compared medical patients with long-standing delirium to outpatients with Alzheimer's disease (AD) and found no significant differences between the two groups. S100B has also been studied in relation to dementia, however without any clear conclusion $[11,20]$.

The CSF biomarkers $\beta$-amyloid 1-42 (A 342 ), total tau (T-tau), and phosphorylated tau (P-tau) are believed to reflect AD brain pathology. Changes in these biomarkers precede the clinical manifestations of dementia by years [21]. For $A \beta 42$, a preclinical phase of up to 20-30 years has been reported [22], while changes in T-tau and P-tau occur later [23]. T-tau is suggested as a marker of neuronal damage and degeneration, and a transient increase has been described in acute stroke [24]. In contrast, P-tau is considered a more specific biomarker for the phosphorylation state of tau, and no significant increase related to acute stroke has been seen [24]. An interaction between S100B and tau has been suggested in previous studies $[25,26]$, possibly with S100B inducing hyperphosphorylation [27]. Our group recently showed that a reduction in CSF A 342 together with an increase in T-tau is associated with an increased risk of delirium in hip fracture patients without dementia, which could indicate that asymptomatic brain pathology has clinical consequences [28].

To elucidate further aspects of the pathophysiology of delirium, the aim of this study was to explore any differences in levels of S100B in CSF and serum of hip fracture patients with prevalent delirium versus never delirium and incident delirium versus never delirium, including stratifications based on clinical dementia and CSF markers of AD. 


\section{Methods}

\section{Hip Fracture Patients}

A total of 332 hip fracture patients were recruited to the Oslo Orthogeriatric Trial at Oslo University Hospital, from September 2009 through January 2012, as described previously $[29,30]$. All patients with proximal femur fractures were suitable for inclusion, regardless of age, prefracture function, cognitive status, and accommodation (including nursing home residents). Individuals were excluded if the fracture was the result of a high-energy trauma or if the patient was considered terminally ill. The orthopedic surgeon on call enrolled the participants in the emergency room. The patients were randomized to treatment in either the orthopedic or the geriatric ward. This intervention had no effect on the incidence of delirium, and patients from both study arms were pooled in the present study.

All patients were assessed for delirium by the study physician (Leiv Otto Watne) or a study nurse using the Confusion Assessment Method [31] daily preoperatively and until the fifth postoperative day (all) or until discharge (delirious patients). Confusion Assessment Method scores were based on information from nurses, close relatives, and hospital records, in combination with a 10- to 30-min interview with the participant. The tests of cognition, attention, and alertness used in the delirium assessments were digit span forward and backward and orientation and delayed recall from the Memorial Delirium Assessment Scale (MDAS) [32]. Patients were classified as "prevalent delirium" (i.e., ongoing delirium at the time of CSF sampling), "incident delirium" (i.e., no delirium at the time of CSF sampling, but developed delirium postoperatively), or "never delirium" (i.e., no delirium throughout the stay). Using all available data (including the Informant Questionnaire on Cognitive Decline in the Elderly [IQCODE] [33], charts, previous cognitive tests [if available], and results from clinical follow-up), two specialists (one geriatrician [Torgeir Bruun Wyller] and one geriatric psychiatrist [Knut Engedal]) reached a consensus as to whether the patients fulfilled the ICD-10 criteria for dementia before their hip fracture [30].

\section{Elective Surgery Patients}

A cohort of elective surgery patients (undergoing gynecological, urological, or orthopedic surgery) was recruited from February 2012 to June 2013 (at Oslo University Hospital and Diakonhjemmet Hospital, Oslo). They underwent thorough cognitive testing before surgery, and patients with known or suspected brain disease were excluded (dementia or Mini-Mental State Examination score [34, 35] $<28$, clock-drawing test score [36] $<4,>1$ false-positive response on the ten-word test from the Consortium to Establish a Registry for Alzheimer's Disease [37], previous stroke with sequelae, Parkinson disease, or other CNS disorders likely to affect cognition). After excluding individuals $<70$ years old or undergoing cancer treatment, 50 elderly patients were included, all free of delirium and dementia at the time of sampling.

\section{Samples, Measurements, and Procedures}

Blood was collected preoperatively (both cohorts) and once postoperatively (hip fracture patients; $2-5$ days after surgery), and serum was stored at $-80^{\circ} \mathrm{C}$. CSF was collected in both cohorts at the onset of spinal anesthesia before administration of the anesthetic agent [29, 30]. CSF was centrifuged as soon as possible and the supernatant was stored at $-80^{\circ} \mathrm{C}$. CSF was thawed, aliquoted, and frozen again before it was sent for analysis of A 342 and P-tau. As patients were included at all hours of the day and all days of the week, CSF and serum samples were collected when logistically feasible. For this reason, all sample types were not available from all cases (Fig. 1). 


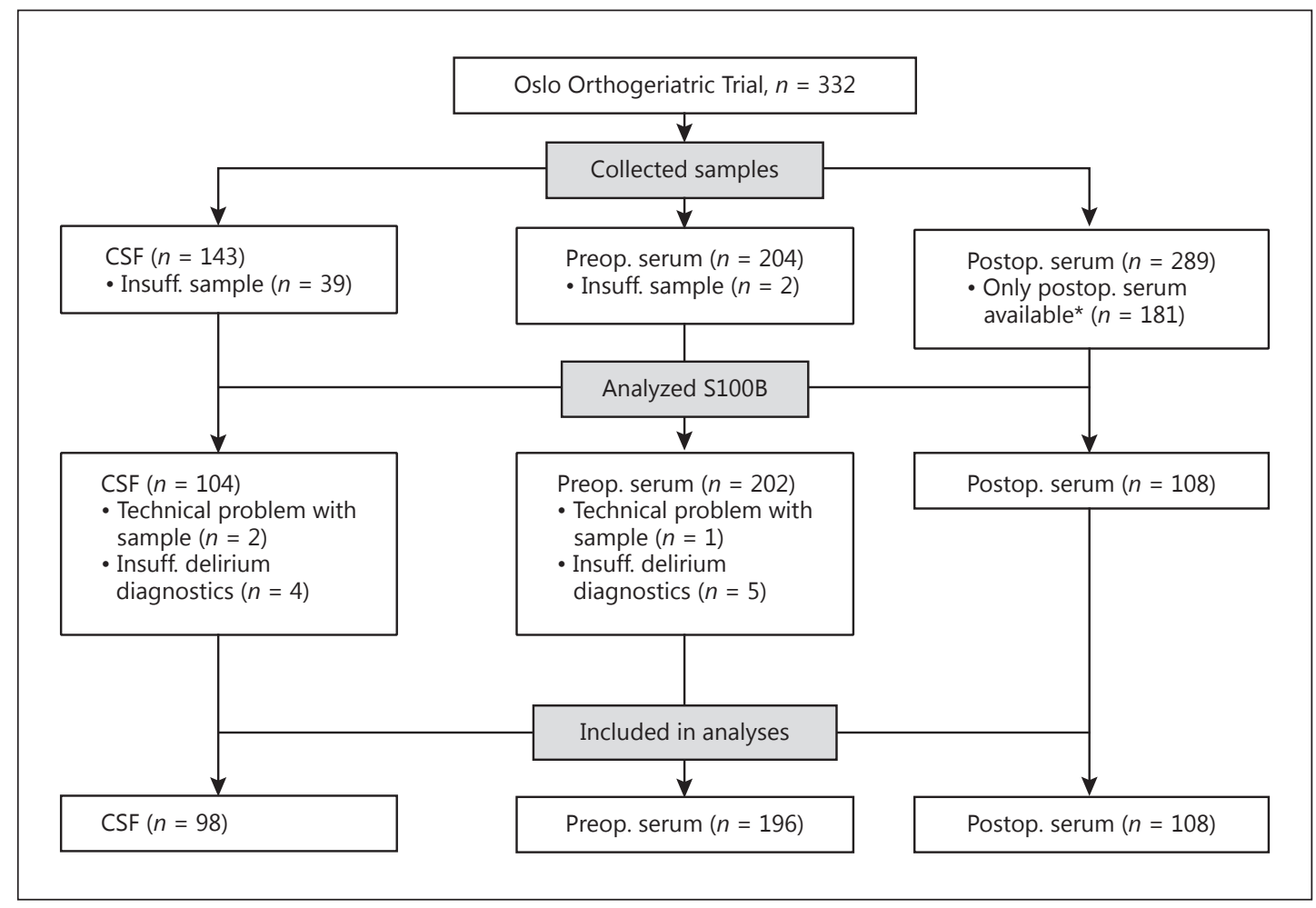

Fig. 1. Study flowchart. * All available samples of CSF and preoperative serum were used, but only postoperative serum in patients with either CSF or preoperative serum. CSF, cerebrospinal fluid.

S100B in serum and CSF was measured using the electrochemiluminescence immunoassay method on the cobas e 601 instrument (Roche Diagnostics, Mannheim, Germany) at Oslo University Hospital, Radiumhospitalet, Norway. A $\beta 42$ and P-tau concentrations were determined using INNOTEST enzyme-linked immunosorbent assays (Fujirebio, Ghent, Belgium) at the Clinical Neurochemistry Laboratory at Sahlgrenska University Hospital, Mölndal, Sweden, performed in one batch of reagents with intra-assay coefficients of variation $<10 \%$. We applied the cutoff values suggested by Hansson et al. [38] with $A \beta 42$ $<530 \mathrm{ng} / \mathrm{L}$ indicating amyloid pathology and P-tau $\geq 60 \mathrm{ng} / \mathrm{L}$ indicating P-tau pathology. We chose P-tau (over T-tau) because it is a more specific biomarker for the phosphorylation state of tau and assumed to be more stable in an acute setting.

\section{Statistical Methods}

Continuous variables were analyzed with the $t$ test (unpaired or paired) or the MannWhitney U test and subgroup differences with ANOVA or the Kruskal-Wallis test, as appropriate. For continuous, nonnormally distributed variables, results are given as median values with interquartile range (IQR), but when possible log-transformed for statistical tests. Correlations were estimated with Spearman's $\rho$.

One case without delirium or dementia had an S100B CSF concentration $>9$ standard deviations from the mean in repeated analyses. The cause is unknown and a technical problem with the handling of this sample may have occurred. This case was excluded from all analyses. 
Table 1. Background characteristics of the hip fracture patients by delirium status

\begin{tabular}{|c|c|c|c|c|}
\hline & $\begin{array}{l}\text { Never delirium } \\
(n=46)\end{array}$ & $\begin{array}{l}\text { Prevalent delirium } \\
(n=36)\end{array}$ & $\begin{array}{l}\text { Incident delirium } \\
(n=16)\end{array}$ & $p$ value $^{\mathrm{a}}$ \\
\hline Median age, years (range) & $84(57-94)$ & $85(68-95)$ & $88(76-101)$ & 0.098 \\
\hline Male, $n(\%)$ & $10(22)$ & $9(25)$ & $7(44)$ & 0.23 \\
\hline Dementia $^{\mathrm{b}}, n(\%)$ & $10(22)$ & $29(81)$ & $10(63)$ & $<0.001$ \\
\hline Independent in $\mathrm{ADL}^{\mathrm{c}}, n(\%)$ & $27(60)^{\mathrm{d}}$ & $8(22)$ & $6(38)$ & 0.002 \\
\hline Living in an institution, $n(\%)$ & $10(22)$ & $19(58)$ & $8(50)$ & 0.008 \\
\hline APACHE II ${ }^{\mathrm{e}}$, median (IQR) & $8.0(6.8-10.0)$ & $9.0(8.0-11.0)$ & $8.0(6.0-9.0)$ & 0.006 \\
\hline Charlson Comorbidity Index, median (IQR) & $0(0-2)^{\mathrm{d}}$ & $1(0-2)$ & $1.0(0-2)$ & 0.070 \\
\hline
\end{tabular}

ADL, activities of daily living; APACHE II, Acute Physiology and Chronic Health Evaluation II; IQR, interquartile range. ${ }^{\mathrm{a}} p$ values for differences in delirium status subgroups (ANOVA or Kruskal-Wallis test, as appropriate). ${ }^{\mathrm{b}}$ Consensus in an expert panel. ${ }^{\mathrm{c}}$ Barthel $\mathrm{ADL} \geq 19 .{ }^{\mathrm{d}}$ Information missing for 1 patient. ${ }^{\mathrm{e}}$ Arterial blood gas and hematocrit omitted from the formula (because not analyzed in all patients).

Table 2. S100B values $(\mu \mathrm{g} / \mathrm{L})$ by delirium status

\begin{tabular}{|c|c|c|c|c|c|c|}
\hline & \multirow{2}{*}{$\begin{array}{l}\text { Elective surgery } \\
\text { patients }\end{array}$} & \multicolumn{5}{|c|}{ Hip fracture patients } \\
\hline & & never delirium & prevalent delirium & $p$ value ${ }^{\mathrm{a}}$ & incident delirium & $p$ value $^{\mathrm{b}}$ \\
\hline \multicolumn{7}{|l|}{ Serum } \\
\hline \multirow[t]{2}{*}{ Preoperative } & $n=50$ & $n=97$ & $n=67$ & 0.33 & $n=32$ & 0.073 \\
\hline & $0.05(0.04-0.06)$ & $0.11(0.07-1.7)$ & $0.11(0.08-0.21)$ & & $0.13(0.09-0.21)$ & \\
\hline \multirow[t]{2}{*}{ Postoperative } & NA & $n=59$ & $n=36$ & 0.85 & $n=22$ & 0.61 \\
\hline & & $0.07(0.05-0.10)$ & $0.07(0.06-0.12)$ & & $0.08(0.05-0.12)$ & \\
\hline \multicolumn{7}{|l|}{$C S F$} \\
\hline \multirow[t]{2}{*}{ All patients } & $n=50$ & $n=46$ & $n=36$ & 0.92 & $n=16$ & 0.013 \\
\hline & 1.09 (0.88-1.38) & $1.08(0.92-1.28)$ & $1.11(0.91-1.29)$ & & $1.38(1.08-1.62)$ & \\
\hline \multirow[t]{2}{*}{ Dementia } & NA & $n=10$ & $n=29$ & 0.21 & $n=10$ & 0.015 \\
\hline & & $1.02(0.69-1.20)$ & $1.13(0.93-1.34)$ & & $1.38(1.06-1.61)$ & \\
\hline \multirow[t]{2}{*}{ No dementia } & $n=50$ & $n=36$ & $n=7$ & 0.25 & $n=6$ & 0.14 \\
\hline & $1.09(0.88-1.38)$ & $1.09(0.92-1.31)$ & $0.95(0.87-1.13)$ & & 1.31 (1.06-1.65) & \\
\hline \multirow[t]{2}{*}{ P-tau $\geq 60$ ng/L } & $n=19$ & $n=17$ & $n=16$ & 0.33 & $n=8$ & 0.002 \\
\hline & $1.15(1.01-1.40)$ & $1.05(0.95-1.16)$ & $1.11(0.97-1.33)$ & & 1.41 (1.11-1.65) & \\
\hline \multirow[t]{2}{*}{ P-tau <60 ng/L } & $n=30$ & $n=27$ & $n=20$ & 0.64 & $n=6$ & 0.68 \\
\hline & $0.98(0.86-1.36)$ & $1.14(0.80-1.32)$ & $1.10(0.86-1.29)$ & & 1.21 (0.95-1.52) & \\
\hline
\end{tabular}

S100B values are presented as median (interquartile range). Bold values denote statistical significance. CSF, cerebrospinal fluid; NA, not available; P-tau, phosphorylated tau. ${ }^{a}$ Never delirium versus prevalent delirium. ${ }^{\mathrm{b}}$ Never delirium versus incident delirium.

\section{Results}

From the Oslo Orthogeriatric Trial, CSF S100B was available from 98 hip fracture patients, preoperative serum S100B from 196 patients, and postoperative serum S100B from 108 patients (Fig. 1). The background data for the hip fracture patients are given in Table 1 . The healthy elective surgery patients $(n=50)$ were younger (72 vs. 85 years, $p<0.001)$ than the hip fracture patients. S100B concentration in serum was significantly higher in hip fracture patients (also in those free from delirium) compared to the elective surgery patients $(p<$ 0.001) (Fig. 2a). In the hip fracture patients, serum concentrations of S100B dropped signifi- 


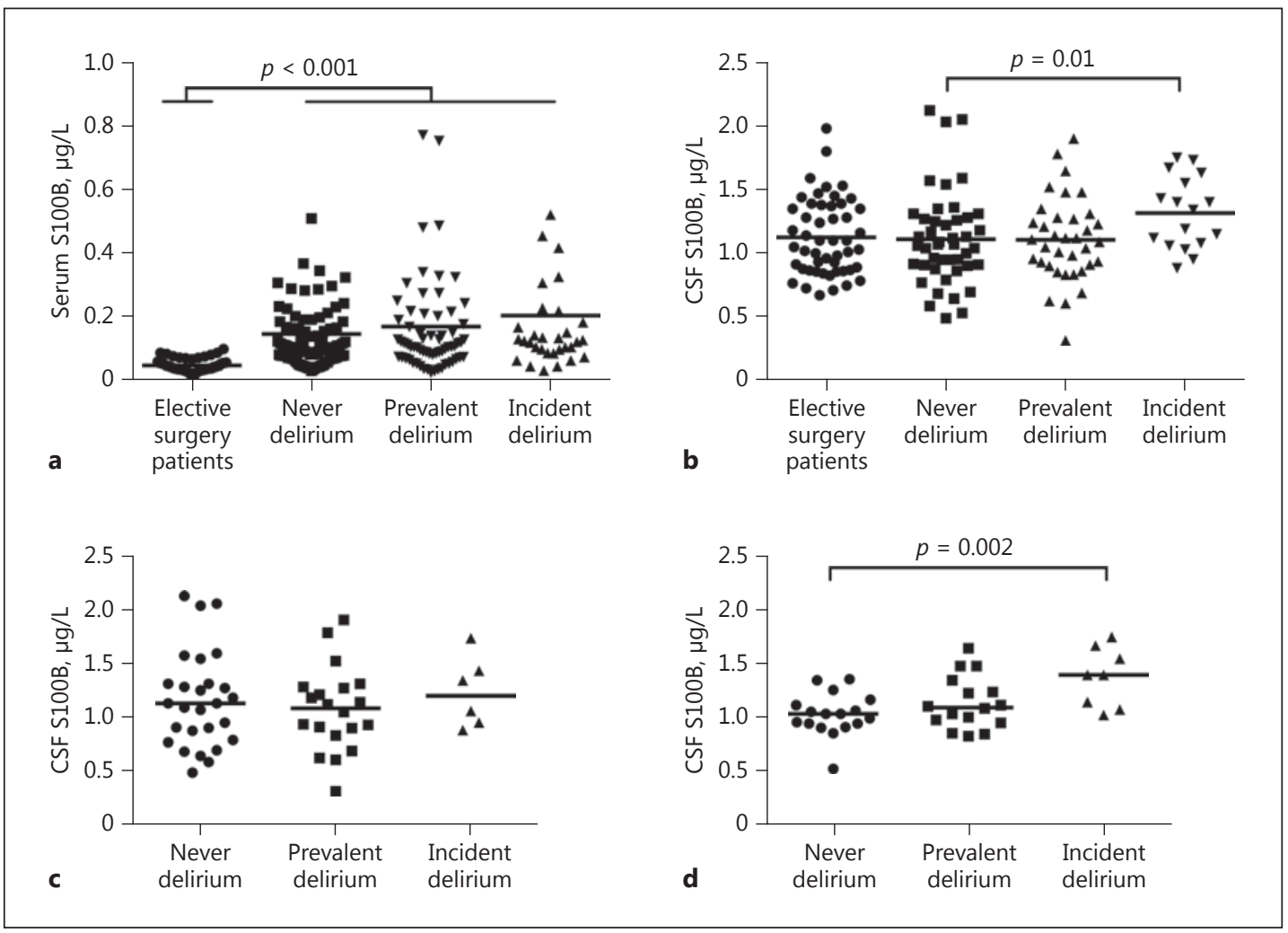

Fig. 2. a Preoperative serum S100B concentration by delirium status. Three outliers had values above the upper margin of this figure: 2.13 (never delirium), 1.52 (incident delirium), and 1.07 (prevalent delirium). b CSF S100B concentration by delirium status. c CSF S100B concentration by delirium status in patients without P-tau pathology (P-tau pathology defined as P-tau $\geq 60 \mathrm{ng} / \mathrm{L}$ ). P-tau was missing in 4 patients. Never delirium $n=27$, prevalent delirium $n=20$, incident delirium $n=6$. d CSF S100B concentration by delirium status in patients with P-tau pathology (P-tau pathology defined as P-tau $\geq 60 \mathrm{ng} / \mathrm{L}$ ). P-tau was missing in 4 patients. Never delirium $n=17$, prevalent delirium $n=16$, incident delirium $n=8$. CSF, cerebrospinal fluid; P-tau, phosphorylated tau.

cantly postoperatively ( $p<0.0001$, paired $t$ test). S100B concentrations in CSF were not significantly different in the group of hip fracture patients as a whole compared to the elective surgery patients $(p=0.66)$. The S100B values are given in Table 2 .

\section{S100B and Delirium}

In the hip fracture cohort, a three-way analysis comparing levels of CSF S100B in patients who never had delirium, patients with prevalent delirium, and patients with incident delirium revealed a significant difference between the groups $(p=0.036)$. A further exploration of this found no significant difference in CSF concentrations of S100B between patients with prevalent delirium and patients who never had delirium ( $p=0.92$ ) (Table 2). Also, there was no significant difference in serum S100B preoperatively between these two groups ( $p=0.33$ ) (Table 2). CSF S100B was however significantly higher in patients with incident delirium than in patients who never developed delirium $(p=0.013)$, and a similar trend was found in serum collected preoperatively ( $p=0.073$ ) (Table 2 ; Fig. 2a, b). As the patients with incident delirium were slightly older than those who never developed delirium (median age 88 [range 76$101]$ vs. 84 [57-94], $p=0.043$ ), we did a regression analysis to adjust for the effect of age (Table 3), yielding a $p$ value of 0.06 but principally the same effect estimates. 
Table 3. Logistic regression models with incident delirium as the dependent variable $(n=62)$

\begin{tabular}{lllllllll}
\hline & \multicolumn{2}{l}{ Unadjusted model } & & & \multicolumn{2}{l}{ Adjusted model } \\
\cline { 2 - 3 } & OR & $95 \%$ CI & $p$ value & & OR & $95 \%$ CI & $p$ value \\
\hline Age, years & 1.07 & $1.03-1.12$ & 0.001 & & 1.10 & $1.00-1.215$ & 0.04 \\
CSF S100B & 5.73 & $1.06-30.91$ & 0.04 & & 5.59 & $0.94-33.11$ & 0.06 \\
\hline
\end{tabular}

CSF, cerebrospinal fluid.

CSF S100B in patients with incident delirium was also higher than in patients in the elective surgery cohort ( $p=0.015$ ) (Table 2). CSF and serum S100B were only correlated in hip fracture patients with delirium $(\rho=0.35, p=0.038)$, but not in those without delirium $(\rho=0.19, p=0.32)$ or in the elective surgery group $(\rho=0.092, p=0.53)$.

\section{S100B in Delirium Stratified on Dementia Status}

Since dementia is very common in this population and a major risk factor for delirium, we repeated the analyses stratified on dementia status. The findings did not materially change; the levels of S100B were still highest in the group with incident delirium (Table 2). Moreover, in the hip fracture group, there was no difference in CSF S100B between patients with dementia ( $n=49$, median $1.13 \mu \mathrm{g} / \mathrm{L}$, IQR 0.93-1.36) and patients without dementia $(n=49$, median $1.10 \mu \mathrm{g} / \mathrm{L}$, IQR $0.93-1.31)(p=0.85)$. There were also no differences in preor postoperative serum values.

\section{S100B in Delirium Stratified on CSF Markers of AD Pathology}

To investigate the relationship between markers of preclinical AD pathology and S100B, we first examined the 50 elective surgery patients, who were all free from dementia and delirium. There was a weak but significant correlation between S100B and P-tau $(\rho=0.35$, $p=0.015)$ in CSF, but no correlation between S100B and A $\beta 42(\rho=0.074, p=0.61)$. Since S100B and P-tau were correlated, we hypothesized that the relationship between S100B and delirium might differ in the groups with and without pathological concentrations of P-tau. Splitting our hip fracture cohort on the presence of P-tau pathology ( $\geq 60 \mathrm{ng} / \mathrm{L}$ ), we found that the difference in CSF S100B between patients with incident delirium, compared to those who never developed delirium, was mainly confined to patients with P-tau pathology (Fig. 2c, d). For $A \beta 42$, only 3 patients without pathological levels $(<530 \mathrm{ng} / \mathrm{L})$ developed delirium at any time, precluding comparable stratification based on $\mathrm{A} \beta 42$.

\section{Discussion}

In this study of S100B in CSF and serum in patients admitted for acute or elective surgery, we did not find elevated concentrations of S100B in CSF during prevalent delirium, unlike one previous study [17]. The elevated S100B in patients developing delirium may however point to pathogenetic mechanisms influencing this condition. Interestingly, elevated S100B seemed to be confined to the group with coexisting pathological changes in the core AD CSF biomarker P-tau, possibly reflecting preexisting pathology of both astrocytic activation (S100B) and neurofibrillary tangles (P-tau), and indicating that these two deviations, in combination, may increase the susceptibility to developing delirium. 
Comparable data are only available in the studies by Hall et al. [17] and Beishuizen et al. [18]. Neither of these studies observed elevated CSF S100B in incident delirium like we did, but Hall et al. found higher concentrations of CSF S100B in patients with prevalent delirium. The reasons for the inconsistencies are not clear; however, the study populations were different, with more nursing home patients and patients with dementia included in our study.

Elevated levels of S100B have previously been considered a marker of acute brain damage [11], and Hall et al. [17] suggest that the elevated CSF S100B levels they observed in prevalent delirium might represent CNS damage or dysfunction related to the episode of delirium. On the other hand, our finding of elevated S100B in incident delirium rather suggests this as indicative of a preexisting process representing a risk factor for delirium development. These hypotheses are not mutually exclusive; S100B may both be an indicator of underlying pathology and in certain situations a marker of damage or dysfunction.

Previous studies of S100B in CSF in AD and dementia have also yielded conflicting results. Comparable to our results, a study of 159 patients with various types of dementia could not find any difference in concentrations of S-100 in dementia patients compared to controls [39]. In contrast, another study found higher concentrations in early/mild AD patients compared to moderate/severe $\mathrm{AD}$ patients and controls [40], suggesting a rise and fall throughout the progression of AD. A correlation has also been reported between S100B concentrations and development of brain atrophy in patients with AD [41]. Specifically, it has been suggested that S100B may play a role in tau-related pathways involved in AD pathology $[25,26]$. Our study supports this hypothesis.

We chose to use a healthy elective surgery cohort to study the relationship between S100B and P-tau unbiased by hip fracture, and found a weak but significant correlation in CSF concentrations. Furthermore, in the hip fracture population, the relationship between S100B and delirium differed in patients with and without P-tau pathology. As the levels of P-tau in CSF are not expected to change in the acute setting [24], it is most likely a marker of underlying pathology. Thus, either differences in prefracture P-tau pathology might cause different S100B responses after hip fracture, or S100B and astrocytic activation may be part of an underlying process also involving P-tau that makes the brain vulnerable to delirium. Caplan et al. [19] did not find differences in S100B in CSF between patients with long-standing delirium and patients with $\mathrm{AD}$; one explanation might be that the patients with $\mathrm{AD}$ also had slightly elevated concentrations of S100B compared to persons with healthy brains. Delirium superimposed on early-stage dementia might have neuropathological processes different from delirium superimposed on late-stage dementia or occurring in cognitively intact patients. It is also possible that S100B levels are affected both by underlying and acute changes. This might result in different CSF S100B patterns in delirium populations with differences in preexisting cognitive impairment and could, in part, explain the different findings between the present and previous studies.

Changes in P-tau pathology are clearly linked to dementia [21]. In our material, however, using a clinical diagnosis of dementia did not reveal the interplay between delirium and AD pathology as clearly as a P-tau cutoff. The levels of S100B in incident delirium were at a similar level in both the dementia and nondementia strata, even thought the difference between incident and never delirium remained significant only in the stratum with dementia. In a larger-scaled study, these subgroup differences might be further explored. It is possible that stratification based on clinical dementia status could reveal the same pattern as we found when stratifying by P-tau. However, it is also possible that the molecular marker P-tau identifies a more homogeneous population of patients with similar underlying disease processes than the clinical diagnosis of dementia. We therefore believe that our approach of stratifying patients based on the presence of biomarkers of neuropathology is a strength of this study and should be utilized in other studies of delirium. 
Hip fracture patients without delirium did not have higher CSF concentrations of S100B than patients in the elective surgery cohort, suggesting that CSF S100B does not necessarily increase solely from a hip fracture. Serum levels, on the other hand, were higher in all groups of hip fracture patients compared to the preoperative levels of elective surgery patients, and the levels dropped significantly after hip fracture surgery. We hypothesize that this represents a non-CNS component in serum S100B in hip fracture patients, independent of delirium, consistent with extracranial sources (i.e., the fracture) and the postoperative value representing a fall towards normal values. This suggests that serum S100B is of limited value as a marker of CNS-related pathology in a hip fracture population.

In the hip fracture population, CSF is easily available from the onset of spinal anesthesia, and the use of CSF biomarkers is of high interest in research to help the understanding of pathophysiological mechanisms. We do, however, not suggest the use of CSF S100B and P-tau for predicting delirium in the clinical setting, as the test's sensitivity and specificity are not good enough. In real life, all hip fracture patients should be considered at risk for delirium and be managed with good care accordingly.

The major strengths of this study are the fairly large sample of CSF in patients with thorough delirium diagnostics combined with repeated serum measures. Still, for subgroup analyses and stratifications, the sample size is a challenge and these findings should be considered explorative. The external group of cognitively healthy elective surgery patients adds further value to the study. Due to the challenges of performing clinical studies of acute patients, the subgroups with serum and CSF samples varied in size, and for some patients only a CSF or only a serum sample was available. However, the background characteristics were similar within the different subgroups (prevalent/incident/no delirium), and the selection of patients was dependent on circumstantial factors (i.e., time of day/week for inclusion/ surgery) and not patient characteristics, decreasing the risk of systematic bias. The study included frail patients and patients with severe dementia. While this leads to a challenging heterogeneity, our observations represent the situation in real life.

Due to the complexity of delirium, future studies would benefit from looking at more than one biomarker, e.g., combining biomarkers for delirium with biomarkers for dementia representing underlying pathology. This calls for larger study samples and could be a great opportunity for large-scale biobanking for delirium research. Also, taken together with previous conflicting results regarding serum S100B, our results suggest that further studies of serum S100B and delirium should not be a priority.

\section{Conclusion}

The main findings of this study were that even though we did not find elevated concentrations of S100B in CSF during prevalent delirium, it was elevated in patients with incident delirium. This finding seemed to be confined to patients with pathological levels of the AD biomarker P-tau, suggesting vulnerability caused by a preexisting process of astrocytic activation and tau pathology.

\section{Acknowledgments}

The authors wish to thank the patients and staff at the Departments of Anesthesiology, Geriatric Medicine, Gynecology, Orthopedic Surgery, and Urology at Oslo University Hospital and the Department of Anesthesiology and the Orthopedic Department at Diakonhjemmet Hospital, Norway. They also thank Professor Emeritus Knut Engedal, Professor Anette Hylen 
Ranhoff, anesthesiologists Randi Støen, Gry Torsæter Dahl, and Arne Myklebust, and research nurses Elisabeth Fragaat, Tone Fredriksen, Camilla Marie Andersen, Julie Ask Ottesen, and Linda Feldt.

\section{Statement of Ethics}

The study was undertaken in accordance with the Declaration of Helsinki. The data and serum/CSF samples were collected after informed consent from the patient and/or proxy (if the patient was lacking the capacity to consent due to delirium and/or dementia), as approved by the Regional Committee for Ethics in Medical and Health Research (South-East Norway) (REK S-09169 and REK 2011/2052).

\section{Disclosure Statement}

Dr. Zetterberg reports that he is a cofounder of Brain Biomarker Solutions in Gothenburg $\mathrm{AB}$, a GU Venture-based platform company at the University of Gothenburg. Dr. Blennow reports personal fees from Fujirebio Europe, IBL International, and Roche Diagnostics, outside the submitted work. The other authors have no disclosures to report.

\section{Funding Sources}

The Oslo Orthogeriatric Trial was mainly funded by the Research Council of Norway through the program "Improving mental health of older people through multidisciplinary efforts" (grant No. 187980/H10). We also received funding from Oslo University Hospital, the Sophies Minde Foundation, the Norwegian Health Association (ID 1513), the Norwegian Association for Public Health, Civitan's Research Foundation, the Medical Student Research Program at the University of Oslo, the Swedish Research Council, the Swedish Alzheimer Foundation, the Swedish Brain Foundation, the Torsten Söderberg Foundation, and the South-Eastern Norway Regional Health Authority. The sponsors had no role in the design, methods, subject recruitment, data collection, analysis, or preparation of the paper.

\section{Author Contributions}

T.B.W. and L.O.W. initiated the study. T.B.W., L.O.W., and K.R.H. designed the study. L.O.W. was responsible for collecting data in the Oslo Orthogeriatric Trial together with F.F. and J.R., while A.V.I. was responsible for collecting data in the elective surgery cohort. N.B. and K.R.H. had responsibility for planning and performing the analyses of S100B in CSF and plasma samples. L.O.W. and A.V.I. were responsible for preparing samples of CSF and sending them to H.Z. and K.B., who were responsible for analyses of amyloid beta and P-tau. K.R.H. and L.O.W. were responsible for statistical analysis. K.R.H., together with L.O.W., T.B.W., and F.A.C., was responsible for interpreting the data and preparing the manuscript, but all authors contributed significantly to this process. 


\section{References}

1 Ryan DJ, O'Regan NA, Caoimh RO, et al: Delirium in an adult acute hospital population: predictors, prevalence and detection. BMJ Open 2013;3:e001772.

2 Witlox J, Eurelings LS, de Jonghe JF, et al: Delirium in elderly patients and the risk of postdischarge mortality, institutionalization, and dementia: a meta-analysis. JAMA 2010;304:443-451.

-3 Inouye SK, Westendorp RG, Saczynski JS: Delirium in elderly people. Lancet 2014;383:911-922.

4 Maclullich AM, Ferguson KJ, Miller T, et al: Unravelling the pathophysiology of delirium: a focus on the role of aberrant stress responses. J Psychosom Res 2008;65:229-238.

5 Ahmed S, Leurent B, Sampson EL: Risk factors for incident delirium among older people in acute hospital medical units: a systematic review and meta-analysis. Age Ageing 2014;43:326-333.

-6 Davis DH, Muniz Terrera G, Keage H, et al: Delirium is a strong risk factor for dementia in the oldest-old: a population-based cohort study. Brain 2012;135:2809-2816.

7 Fong TG, Jones RN, Shi P, et al: Delirium accelerates cognitive decline in Alzheimer disease. Neurology 2009; 72:1570-1575.

-8 Davis DH, Muniz-Terrera G, Keage HA, et al: Association of delirium with cognitive decline in late life: a neuropathologic study of 3 population-based cohort studies. JAMA Psychiatry 2017;74:244-251.

-9 Hachem S, Aguirre A, Vives V, et al: Spatial and temporal expression of S100B in cells of oligodendrocyte lineage. Glia 2005;51:81-97.

10 Vives V, Alonso G, Solal AC, et al: Visualization of S100B-positive neurons and glia in the central nervous system of EGFP transgenic mice. J Comp Neurol 2003;457:404-419.

11 Michetti F, Corvino V, Geloso MC, et al: The S100B protein in biological fluids: more than a lifelong biomarker of brain distress. J Neurochem 2012;120:644-659.

12 van Munster BC, Korevaar JC, Korse CM, et al: Serum S100B in elderly patients with and without delirium. Int J Geriatr Psychiatry 2010;25:234-239.

13 van Munster BC, Korse CM, de Rooij SE, et al: Markers of cerebral damage during delirium in elderly patients with hip fracture. BMC Neurol 2009;9:21.

14 Herrmann M, Ebert AD, Galazky I, et al: Neurobehavioral outcome prediction after cardiac surgery: role of neurobiochemical markers of damage to neuronal and glial brain tissue. Stroke 2000;31:645-650.

15 Beishuizen SJ, Scholtens RM, van Munster BC, et al: Unraveling the relationship between delirium, brain damage, and subsequent cognitive decline in a cohort of individuals undergoing surgery for hip fracture. J Am Geriatr Soc 2017;65:130-136.

16 Unden J, Bellner J, Eneroth M, et al: Raised serum S100B levels after acute bone fractures without cerebral injury. J Trauma 2005;58:59-61.

17 Hall RJ, Ferguson KJ, Andrews M, et al: Delirium and cerebrospinal fluid S100B in hip fracture patients: a preliminary study. Am J Geriatr Psychiatry 2013;21:1239-1243.

18 Beishuizen SJ, Scholtens RM, Vellekoop AE, et al: Timing is critical in determining the association between delirium and S100 calcium-binding protein B. J Am Geriatr Soc 2015;63:2212-2214.

19 Caplan GA, Kvelde T, Lai C, et al: Cerebrospinal fluid in long-lasting delirium compared with Alzheimer's dementia. J Gerontol A Biol Sci Med Sci 2010;65:1130-1136.

-20 Leclerc E, Sturchler E, Vetter SW: The S100B/RAGE axis in Alzheimer's disease. Cardiovasc Psychiatry Neurol 2010;2010:539581.

21 Blennow K, Hampel H, Weiner M, et al: Cerebrospinal fluid and plasma biomarkers in Alzheimer disease. Nat Rev Neurol 2010;6:131-144.

-22 Jansen WJ, Ossenkoppele R, Knol DL, et al: Prevalence of cerebral amyloid pathology in persons without dementia: a meta-analysis. JAMA 2015;313:1924-1938.

23 Jack CR Jr, Knopman DS, Jagust WJ, et al: Tracking pathophysiological processes in Alzheimer's disease: an updated hypothetical model of dynamic biomarkers. Lancet Neurol 2013;12:207-216.

-24 Hesse C, Rosengren L, Andreasen N, et al: Transient increase in total tau but not phospho-tau in human cerebrospinal fluid after acute stroke. Neurosci Lett 2001;297:187-190.

25 Yu WH, Fraser PE: S100beta interaction with tau is promoted by zinc and inhibited by hyperphosphorylation in Alzheimer's disease. J Neurosci 2001;21:2240-2246.

-26 Baudier J, Cole RD: Interactions between the microtubule-associated tau proteins and S100b regulate tau phosphorylation by the $\mathrm{Ca}^{2+}$ /calmodulin-dependent protein kinase II. J Biol Chem 1988;263:5876-5883.

-27 Esposito G, Scuderi C, Lu J, et al: S100B induces tau protein hyperphosphorylation via Dickopff-1 up-regulation and disrupts the Wnt pathway in human neural stem cells. J Cell Mol Med 2008;12:914-927.

28 Idland AV, Wyller TB, Stoen R, et al: Preclinical amyloid- $\beta$ and axonal degeneration pathology in delirium. Journal of Alzheimer's Disease: J Alzheimers Dis 2017;55:371-379.

29 Watne LO, Torbergsen AC, Conroy S, et al: The effect of a pre- and postoperative orthogeriatric service on cognitive function in patients with hip fracture: randomized controlled trial (Oslo Orthogeriatric Trial). BMC Med 2014;12:63.

-30 Wyller TB, Watne LO, Torbergsen A, et al: The effect of a pre- and post-operative orthogeriatric service on cognitive function in patients with hip fracture. The protocol of the Oslo Orthogeriatric Trial. BMC Geriatr $2012 ; 12: 36$ 
31 Inouye SK, van Dyck CH, Alessi CA, et al: Clarifying confusion: the confusion assessment method. A new method for detection of delirium. Ann Intern Med 1990;113:941-948.

32 Breitbart W, Rosenfeld B, Roth A, et al: The Memorial Delirium Assessment Scale. J Pain Symptom Manage 1997;13:128-137.

-33 Jorm AF: A short form of the Informant Questionnaire on Cognitive Decline in the Elderly (IQCODE): development and cross-validation. Psychol Med 1994;24:145-153.

34 Folstein MF, Folstein SE, McHugh PR: "Mini-mental state." A practical method for grading the cognitive state of patients for the clinician. J Psychiatr Res 1975;12:189-198.

-35 Engedal K, Haugen P, Gilje K, et al: Efficacy of short mental tests in the detection of mental impairment in old age. Compr Gerontol A 1988;2:87-93.

36 Shulman KI: Clock-drawing: is it the ideal cognitive screening test? Int J Geriatr Psychiatry 2000;15:548-561.

-37 Morris JC, Heyman A, Mohs RC, et al: The Consortium to Establish a Registry for Alzheimer's Disease (CERAD). Part I. Clinical and neuropsychological assessment of Alzheimer's disease. Neurology 1989;39:1159-1165.

-38 Hansson 0, Zetterberg H, Buchhave P, et al: Association between CSF biomarkers and incipient Alzheimer's disease in patients with mild cognitive impairment: a follow-up study. Lancet Neurol 2006;5:228-234.

-39 Nooijen PT, Schoonderwaldt HC, Wevers RA, et al: Neuron-specific enolase, S-100 protein, myelin basic protein and lactate in CSF in dementia. Dement Geriatr Cogn Disord 1997;8:169-173.

40 Peskind ER, Griffin WS, Akama KT, et al: Cerebrospinal fluid S100B is elevated in the earlier stages of Alzheimer's disease. Neurochem Int 2001;39:409-413.

41 Petzold A, Jenkins R, Watt HC, et al: Cerebrospinal fluid S100B correlates with brain atrophy in Alzheimer's disease. Neurosci Lett 2003;336:167-170. 